\title{
Correction to: Radiographic review of anatomy and pathology of the masticator space: what the emergency radiologist needs to know
}

\author{
Shahzaib Chughtai ${ }^{1} \cdot$ Komal A. Chughtai $^{2}$ (D) $\cdot$ Simone Montoya ${ }^{2} \cdot$ Alok A. Bhatt $^{3}$
}

Published online: 14 March 2020

(C) American Society of Emergency Radiology 2020

\section{Correction to: Emergency Radiology (2020)}

https://doi.org/10.1007/s10140-020-01756-7

Unfortunately, the original publication of this article contained a mistake in Fig. 7a. The correct figure is presented here. The original article has been corrected.

The online version of the original article can be found at https://doi.org/ 10.1007/s10140-020-01756-7

Komal A. Chughtai

komal_chughtai@urmc.rochester.edu

Shahzaib Chughtai

shachu@umich.edu

Simone Montoya

simone_montoya@urmc.rochester.edu

Alok A. Bhatt

Bhatt.Alok@mayo.edu

1 University of Michigan Medical School, 1301 Catherine St, Ann Arbor, MI 48109, USA

2 University of Rochester Medical Center, 601 Elmwood Ave, Rochester, NY 14642, USA

3 Mayo Clinic, 4500 San Pablo Rd S, Jacksonville, FL 32224, USA 

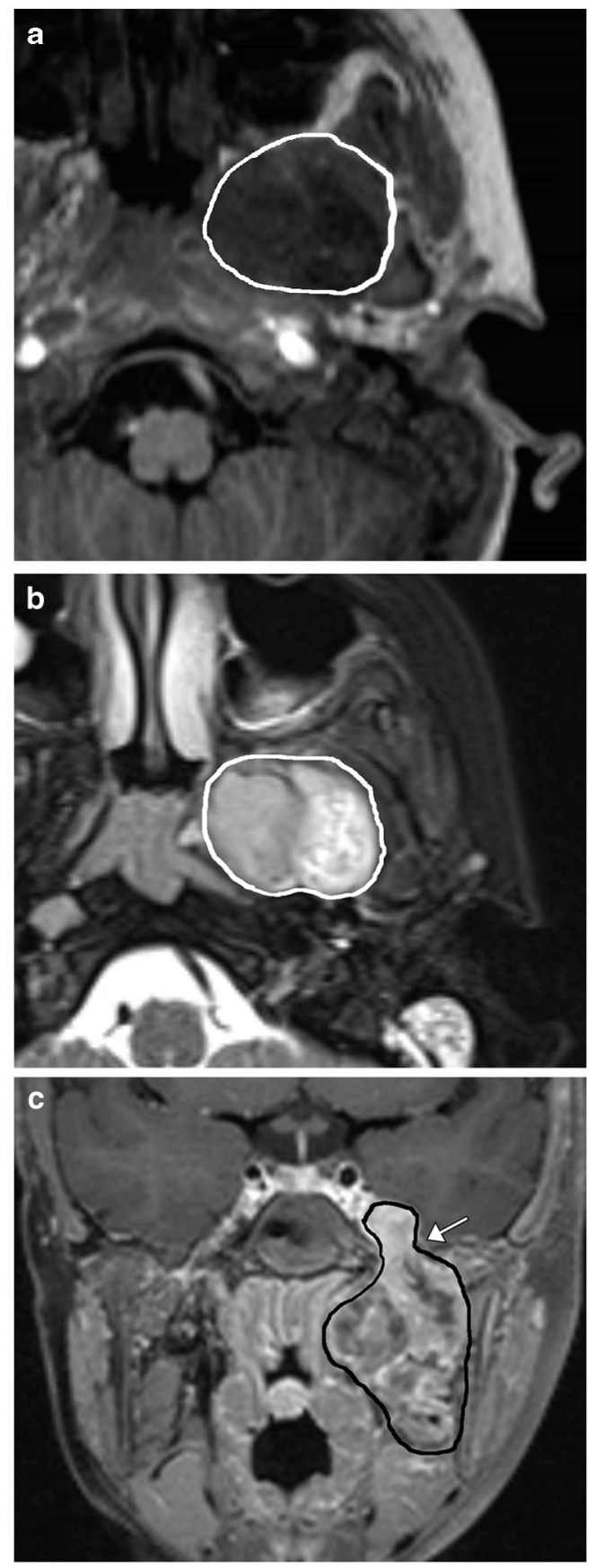

Fig. 7 Rhabdomyosarcoma. Seven-year-old male with left facial mass. a, b Axial T1-weighted and T2-weighted (fat-saturated) images show a well-defined lobulated mass arising from the left medial pterygoid muscle (solid outline), T1-hypointense and T2-hyperintense to normal muscle. Mass effect results in rightward deviation of the nasopharynx and compression of the Eustachian tube. c Coronal T2-weighted image with fat saturation shows the extends superiorly along the mandibular division of the left trigeminal nerve, through the foramen ovale and into the cavernous region

Publisher's note Springer Nature remains neutral with regard to jurisdictional claims in published maps and institutional affiliations. 